\title{
The impact of wind uncertainty on the strategic valuation of distributed electricity storage
}

\author{
Pedro Crespo Del Granado • Stein W. \\ Wallace · Zhan Pang
}

Submitted: February 2014 / Revised: December 2014

\begin{abstract}
The intermittent nature of wind energy generation has introduced a new degree of uncertainty to the tactical planning of energy systems. Shortterm energy balancing decisions are no longer (fully) known, and it is this lack of knowledge that causes the need for strategic thinking. But despite this observation, strategic models are rarely set in an uncertain environment. And even if they are, the approach used is often inappropriate, based on some variant of scenario analysis - what-if analysis. In this paper we develop a deterministic strategic model for the valuation of electricity storage (a battery), and ask: "Though leaving out wind speed uncertainty clearly is a simplification, does it really matter for the valuation of storage?". We answer this question by formulating a stochastic programming model, and compare its valuation to that of its deterministic counterpart. Both models capture the arbitrage value of storage, but only the stochastic model captures the battery value stemming from wind speed uncertainty. Is the difference important? The model is tested on a case from Lancaster University's campus energy system where a wind turbine is installed. From our analysis, we conclude that considering wind speed uncertainty can increase the estimated value of storage with up to $50 \%$ relative to a deterministic estimate. However, we also observe cases where wind speed uncertainty is insignificant for storage valuation.
\end{abstract}

Pedro Crespo Del Granado ${ }^{\mathbf{1}} \cdot$ Zhan Pang $^{\mathbf{2}}$

Energy Science Center, ETH Zürich ${ }^{\mathbf{1}}$

Sonneggstrassse 28, 8092 Zürich, Switzerland. E-mail: pedro@esc.ethz.ch ${ }^{\mathbf{1}}$

Department of Management Science, Lancaster University Management School ${ }^{\mathbf{1 , 2}}$

Lancaster LA1 4YX, United Kingdom. E-mail: z.pang@lancaster.ac.uk²

Stein W. Wallace

Department of Business and Management Science, Norwegian School of Economics NO-5045 Bergen, Norway. E-mail: Stein.Wallace@nhh.no 
Keywords Smart grid · wind energy · energy storage · uncertainty · valuation $\cdot$ stochastic programming

\section{Introduction}

The UK has set a binding target of generating $15 \%$ of its energy demand from renewable sources by 2020 (DECC 2013) while the EU aims to reach a $20 \%$ renewable share by 2020 (European Commission 2014). As the energy generation of renewables depends on exogenous factors (e.g. wind speed for wind power), this creates intermittency in generation and new planning challenges to maintain a stable and reliable supply-demand balance. One technology that has the potential to alleviate the intermittency problem posed by wind generation is energy storage. A battery could provide leverage to shift bulk energy over periods of several hours (e.g. peak-time management) or to smooth renewable output and correlate it with local demand.

Indeed, there has been a resurgence interest in electricity storage because of the necessity to support new distributed generation (DG) clusters of windmills spread out over the country that feed end-users directly (Carbon Connect 2012). For instance, grid operators will increasingly need to synchronize (in real-time) an equivalent reduction in the output of the large central generators. That is, an intermittent load of potential "prosumers" has to be integrated somehow to the already arduous grid supply-demand out-turn operations. As a matter of fact, upgrading the power system for these changes has proved to be challenging and policy commitments have been revised several times to reconsider renewable penetration or to postpone targets in hopes that the system either gets smarter or more flexible. To this effect, the UK's ongoing nationwide roll out of advanced smart metering infrastructure by 2015-2016 has been envisaged as a game changer (Teh et al. 2011). A smart grid network enables active participation by consumers and can transform renewable generation from an energy source into a dispatchable generation source. In this real-time responsive system, electricity storage is the key component for smart grids and now has the long-sought opportunity of unlocking its economic viability. Energy storage not only facilitates the integration of larger quantities of DG to the main grid, but through a smart grid adds flexibility as a demandside management mechanism. This will allow DG systems not to be merely connected to the network, but also integrated into the overall operation of this future power system.

To understand the potential synergies between the end-user DG and storage technologies, in a previous paper (Crespo Del Granado et al., 2014), we analyse these synergies by modelling the energy system of residential buildings. The study focuses on inter-temporal variations of wind power and electricity spot prices, though it ignores wind uncertainty. The numerical results from the model highlight the synergy value between small-scale wind turbines and batteries. As expected, the wind turbine has a significant contribution to the battery and the battery in turn increases the utilization of the wind power. 


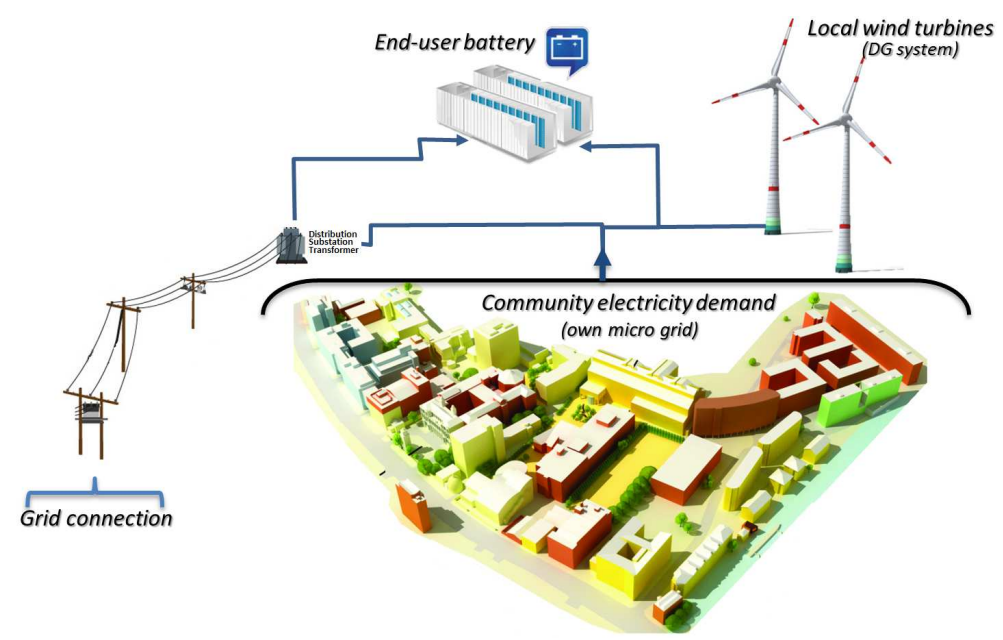

Fig. 1 Schematic diagram of a local-community's distributed generation (wind) in sync with a large battery and the grid.

This can be interpreted by the two-fold role of the storage: as inventory mechanism that responds to demand and supply variations and as arbitrage mechanism that addresses the inter-temporal price variations. The more volatile the demand and supply (wind in this case), the more valuable the storage. The study demonstrates that the integration of wind power and storage technologies can contribute to energy savings for the end-user, smooth the energy consumption from the grid perspective and hence improve energy efficiency.

On the same premise, this paper extends the study of Crespo Del Granado et al. (2014) to a large end-user with its own operational micro grid in sync with an on-site DG system. More specifically, we consider a community's DG system consisting of a combined heat and power $\left(\mathrm{CHP}^{1}\right)$ unit and wind turbines (see Fig. 1 for an illustration of this system). The objective of this community energy system is to minimize the total expected cost of its load commitments to the local utility or grid operator over a finite horizon. Similar setups already exist with some large scale industrial sites that defer high energy consuming manufacturing processes to off-peak price periods. For our case, however, the battery storage induces cost savings from energy arbitrage (smart grid setting) and from smoothing wind fluctuations. As a result, this model can be applied to any price-taking day-ahead participant (market or end-user) who is interested in assessing the economic value of electricity storage. In our model we use dynamic pricing (i.e. wholesale spot prices as proxies for energy cost) as a representative mechanism of real-time grid operations costs.

In this setting, though the short-term intertemporal decisions of storage are modelled, the economic viability of energy storage remains elusive if aspects of wind intermittency are not taken into consideration. The strategic decision of

1 A CHP is a gas driven energy unit that generates electricity and heat simultaneously. 
whether or not electricity storage is worth installing could depend upon how the valuation model captures features of wind uncertainty and its effect on the value of storage. For instance, modelling wind under a perfect foresight assumption (that is, studying the deterministic expected case) might underestimate the flexibility value of energy storage. In this paper, we emphasise modelling features necessary to show whether or not there is a business case for energy storage when a strategic assessment considers wind intermittency.

Here one of the main challenges of wind energy is its dependency on the unpredictability and variability of wind speeds. In this sense, to better measure the effect of wind uncertainty on the value of storage, we model wind by constructing scenarios under a stochastic programming framework. The scenarios are created from a time series analysis which simulates the stochastic realizations of the wind speeds. The model is used to assess the importance of taking wind uncertainty into account when valuing energy storage. As a consequence, our results also show how the proportion of local DG (relative to end-user's demand) affects the economic value of storage and its relationship with wind uncertainty when the decision context becomes stochastic.

Thus, it is important to stress that in this paper we are asking if the value of electricity storage can be well estimated with a deterministic model. This is not the same as estimating the famous value of the stochastic solution (VSS), which rather asks how well the deterministic solution behaves in a stochastic environment. Our concern is that strategic decisions are often made based on simple deterministic modelling which might lead to misguided decisions.

The paper is organized as follows. In Section 2 we discuss existing academic work on modelling the value of energy storage for different applications and perspectives, while in Section 3, we discuss the features of distributed generation systems. Then, in Section 4, we present the multi-stage stochastic programming model under wind uncertainty along with the scenario generation approach. This is followed by the description of an applied case study in Section 5 and the conclusions in Section 6.

\section{Related models for energy storage valuation}

In general, the value of energy storage stems from its contribution to energy arbitrage, power quality, transmission support, and balancing services for renewable energy sources. According to Eyer and Corey (2010) and EPRI (2010) the value of storage can be further divided into 17 to 21 types of application benefits for different power system configurations. As a result, different research standpoints emerge that vary based on storage technologies, modelling assumptions, market-regulatory structure and ownership considerations. In short, the existing literature has covered the grid supply-side perspective, and only since recently a demand-side management mechanism is being studied.

Taking a grid perspective, Sioshansi et al. (2009) explore the sensitivity of price to energy arbitrage for different storage sizes and round-trip efficiencies in the PJM market region. Similarly, Walawalkar et al. (2007) combine arbi- 
trage and frequency regulation applications for the New York grid region to conclude that NaS (Sodium sulphur battery) and flywheel storage technologies are probably worthwhile investments. Similarly, Nyamdash et al. (2010) study the economic viability of different large energy storage technologies in the Irish grid system under current market conditions and the prospects of enhancing wind integration. But their study concludes that storage is not economically feasible since high capacity combinations of wind and storage sometimes displace conventional units, generating more cycling in medium-base load plants. Wind integration issues are extended further by Sundararagavan and Baker (2012) for 11 different storage technologies and their role in different applications. Likewise, Hittinger et al. (2012) identified the most valuable applications for grid energy storage for emerging energy storage technologies. As the value of storage is case-specific in these papers, He et al. (2011) studies a business model for the Belgian grid to analyse the effects of different market schemes on the value of storage arbitrage. Overall, this top-down grid level research tends to ignore the heterogeneity of energy demand and the potential savings for end-users if these are integrated as a flexible demand (i.e. distributed storage presence) as addressed in this paper.

Another important storage literature stream centres on energy suppliers and their energy commitments to deregulated electricity markets. A wellknown application is to maximize profits through optimal scheduling operations of stored hydro-power and wind farms in day-ahead markets (Castronuovo and Lopes 2004). In this literature, based on the problem characteristics, the impact of short-term uncertainty is analysed for demand (Brown et al. 2008), prices (Mokrian and Stephen 2006), and wind generation (Korpaas et al. 2003; Vespucci et al. 2010). In a similar way, attention is paid to modelling the electricity market context (Garcia-Gonzalez et al. 2008), theoretical approaches to computing the value of the storage (Kim and Powell 2011), and the robustness of optimal solutions for different policies/strategies (Scott and Powell 2012). Since studying the value of energy storage is likely to produce a context-specific rather than a general answer, our focus is explicitly on developing a valuation model for customer-site storage with DG. In this paper, we put emphasis on how important the effects of wind uncertainty are on the possible strategic decision of installing a battery for a large end-user.

Indeed, in recent years, understanding the interplay between monitored controllable loads (through energy storage) and new distributed generation technologies is a major area of research in smart grids (Roberts and Sandberg 2011). In a previous study (Crespo Del Granado et al., 2014) we assess the value of deploying lead-acid batteries to UK residential buildings with a smart grid perspective. We found that when electricity storage is combined with dynamic pricing and house demand is taken into account, end-user cost savings were in the order of $7-15 \%$ from the reference case (no storage presence). Additionally, special attention was given to the presence of small wind turbines in households - increasing the value of storage savings to 9-18\%. Similar work by Harsha and Dahleh (2015) considered the effect of wind uncertainty in U.S. dwellings, and reported $7-13 \%$ in cost savings from wind and storage penetra- 
tion. Other studies, such as Ahlert and van Dinther (2009) put an emphasis on price uncertainty and noted savings up to $17 \%$ for different battery sizes in German residential buildings. A more recent study by Mishra et al. (2012), with similar focus, but applied to a different set of American households, ascertains the value of energy storage to be $10-15 \%$.

In contrast, we develop a stochastic optimization model for a community or small town with a medium-sized owned (distributed) generation to focus on the value of storage under uncertain wind conditions. For example, literature on real-life DG units with storage has been limited to study the value of solar PV systems (Hanna et al., 2014), the commercial strategies on supporting distribution network operations (Moreno et al., 2015) and modelling uncertain aspects of the battery technology (Cardoso et al., 2013). Hence, our contribution is to study the value effect of wind uncertainty on the decision to invest in a battery for a large end-user. We are interested in analysing the value of a battery for a self-contained micro grid system with the assumption that no DG surplus is sold back to the grid. This premise is in line with our case study, and will be typical for many micro grids.

\section{Distributed energy systems with electricity storage}

Distributed generation (DG) or decentralized generation refers to delivering power directly to the demand loads. This is in contrast to conventional generation, where network transmission and distribution services are required to deliver power from central generators to end-users. For instance, distributed generation partially backs up the demand from many industrial facilities, building complexes, hospitals or campuses. In the UK, this embedded generation today represents around $11 \%(9 \mathrm{GW})$ of the generating capacity with $55 \%$ of this being renewable (see Carbon Connect 2012).

The distributed generation system considered in this paper consists of a CHP unit and a wind turbine. Their combined energy production approximately covers the user's base load demand. Additional power (see Figure 1) comes from the grid connection. Assuming that a smart grid is in place, we develop a model from the point of view of a large end-user who considers installing a medium-large size electricity storage unit to minimize the grid consumption cost (i. e. energy arbitrage) as well as to smooth renewable generation. As the end-user is interested in cost savings from DG production, we assume that there is no availability to feed-back electricity to the grid.

We employ a multi-stage stochastic linear programming approach to investigate the impact of wind uncertainty on the value of energy storage. A finite planning horizon of one day divided into 48 half-hour discrete time intervals is indexed by $t=1,2, \ldots, 48$. In each time step demand, electricity prices and wind scenarios are fed into the model as time varying parameters. Our model has two main sets of constraints for each time $t$ : a supply-demand inequality constraint and an inventory equality constraint for energy storage operations. Moreover, each unit is subjected to its own operating constraints. 


\subsection{Local micro grid}

A micro grid is a confined electrical distribution network which operates local DG sources and controllable loads autonomously in a single system (Hatziargyriou et al. 2007). In a local micro grid, the energy input-output is in sync, through a coupling transformer and an electric switchgear, with the utility grid's electricity voltage and frequency levels. Moreover, as a self-contained power system, micro grids have an electrical grid architecture consisting of converters/inverter modules attached to generating units and loads. This is used to regulate voltage/frequency within the private distribution network.

An important aspect of smart grids is the grid interaction with the micro grid since local demand is seen as a dispatchable load by the main grid. We address this by a load-balance constraint (supply $\geq$ demand) for each time period $t$. Thus, on one hand, the DG energy system supply has available wind generation noted as $\xi_{\text {wind }}^{(t)}$, electricity consumption from the grid connection $C_{\text {grid }}^{(t)}$, local base load generation $L_{\text {base }}$ and battery output or discharge $B_{\text {out }}^{(t)}$. On the other hand, the demand side loads are the local electricity demand $d_{\text {load }}^{(t)}$ and battery charging $B_{i n}^{(t)}$. This is represented as follows (for $t=1,2, \ldots, 48$ ):

$$
\overbrace{\xi_{\text {wind }}^{(t)}+C_{\text {grid }}^{(t)}+B_{\text {out }}^{(t)}+L_{\text {base }}}^{\text {Wind+Grid+Battery Discharge+Base load }} \geq \overbrace{d_{\text {load }}^{(t)}+B_{\text {in }}^{(t)}}^{\text {Demand+Battery Charge }}
$$

Implicit in this modelling constraint is the assumption that there is a physical way to make supply equal to demand, as of course, in reality supply must equal demand all the time. Hence, we assume that when the left-hand side is larger than the right-hand side, there will be no electricity supplied either from the grid or the battery (this follows from the fact that in optimal solutions, we would never pay and then waste, or discharge from the battery for no reason), and that wind energy can be wasted at no cost. Though not very relevant in our case study, we also assume that if $L_{\text {base }}>d_{\text {load }}^{(t)}$, there is a way to either use that power elsewhere (for example by heating water), or in the extreme case, waste it.

Note that (1) assumes that wind energy $\xi_{\text {wind }}^{(t)}$ is a known deterministic parameter. In the next section, we discuss thoroughly how to model wind uncertainty in our stochastic multi-stage model for the day-ahead planning horizon. The objective is to minimize grid consumption $C_{\text {grid }}^{(t)}$ given that it is exposed to dynamic electricity prices $p_{e l}^{(t)}$ in a smart grid setting (i.e. wholesale spot prices as proxies for energy cost). The notation used throughout the paper is summarized in Table 1.

\subsection{Wind turbines}

Low-carbon incentives for a cleaner energy generation has made wind power one of the most adopted sources for DG. Typically, wind power is generated 
Table 1 Nomenclature used to represent indices, parameters and variables.

\begin{tabular}{|c|c|c|}
\hline Symbol & Definition & \\
\hline $\begin{array}{c}k \in K \\
t \in T_{k} \\
\omega_{k} \in \Omega_{k}\end{array}$ & $\begin{array}{l}\text { K number of stages indexed by } k=1,2, \ldots, K \\
\text { Half hourly time period } t \text { in set } T_{k} \text { at stage } k \\
\text { Wind scenario index in set } \Omega_{k} \text { at stage } k\end{array}$ & Indices \\
\hline $\begin{array}{c}\xi_{\text {wind }}^{\left(t, \omega_{k}\right)} \\
\pi_{\omega_{k}} \\
v^{(t)}\end{array}$ & $\begin{array}{l}\text { Wind energy scenario } \\
\text { Probability of scenario } \xi_{\text {wind }}^{\left(t, \omega_{k}\right)} \text { at stage } k \\
\text { Wind speeds used in the power curve }\end{array}$ & $\begin{array}{l}\text { Stochastic } \\
\text { wind } \\
\text { parameters }\end{array}$ \\
\hline $\begin{array}{c}d_{\text {load }}^{(t)} \\
p_{e l}^{(t)}\end{array}$ & $\begin{array}{l}\text { Local community electricity demand (MWh) } \\
\text { Electricity wholesale spot prices }(£ / M W h)\end{array}$ & Time parameters \\
\hline $\begin{array}{l}C_{g r i d}^{\left(t, \omega_{k}\right)} \\
S^{\left(t, \omega_{k}\right)} \\
B_{i n}^{\left(t, \omega_{k}\right)} \\
B_{\text {out }}^{\left(t, \omega_{k}\right)}\end{array}$ & $\begin{array}{l}\text { Grid electricity consumption to demand/battery } \\
\text { Amount of electricity stored in the battery } \\
\text { Battery charging input from the grid or/\& wind } \\
\text { Battery discharge output to electricity demand }\end{array}$ & Decision variables \\
\hline $\begin{array}{c}\bar{S}_{\max } \\
\underline{S}_{\min } \\
\alpha_{\max } \\
\beta_{\max } \\
\eta_{\text {eff }}\end{array}$ & $\begin{array}{l}\text { Battery upper capacity bound } \\
\text { Battery lower capacity bound } \\
\text { Battery maximum charging rate } \\
\text { Battery maximum discharging rate } \\
\text { Battery round-trip efficiency }\left(\eta_{\text {eff }} \in[0,1]\right)\end{array}$ & Storage parameters \\
\hline$L_{\text {base }}$ & Constant energy inflow from base-load unit & Base-load parameter \\
\hline
\end{tabular}

by extracting the wind speeds' kinetic energy through a wind turbine rotor. Since the energy output is proportional to the wind speed, a common way to represent this relationship is by the so-called wind power curve. This curve is empirically estimated by the rotor manufacturing design rating and to a lesser extent by ambient pressure and temperature. Figure 2 illustrates the power/wind speed curve of a $2.3 \mathrm{MW}$ wind turbine with power output at various speeds levels ${ }^{2}$. Note that no significant power is produced until the cutin speed $(3 \mathrm{~m} / \mathrm{s}$ in this case) followed by an approximately cubic relationship between wind speeds and output power (until the maximum power output).

To represent $\xi_{\text {wind }}^{(t)}$, in our model, we fit a polynomial curve to the output power given a wind speed data-set $\left(v^{(t)}\right)$. As seen in Figure 2, this polynomial ${ }^{3}$ is fitted between the cut-in wind speed and the maximum output at $16 \mathrm{~m} / \mathrm{s}$. Hence, wind speed frequency is also of great importance. Figure 2 also shows a typical annual frequency distribution for 10 minute mean wind speeds from a lowland site in the UK (see Hazelrigg Weather Station). Comparing the power curve and the frequency distribution, we can see that the turbine operates at its rated output $(2300 \mathrm{~kW})$ around $8 \%$ of the year, while the turbine is idle at low winds about $30 \%$ of the time, and during the remaining period the output fluctuates notably in the upward part of the curve. This shows that on top of the challenges related to wind forecasting - a weather related uncertainty

\footnotetext{
2 Wind turbine Model E-70 manufactured by ENERCON GmbH; further technical information available at http://www.enercon. de (accessed on July 31, 2013).

$3 \xi_{\text {wind }}(v)=0.0498 v^{5}-2.5155 v^{4}+44.91 v^{3}-336.68 v^{2}+1165.9 v-1480.5$
} 


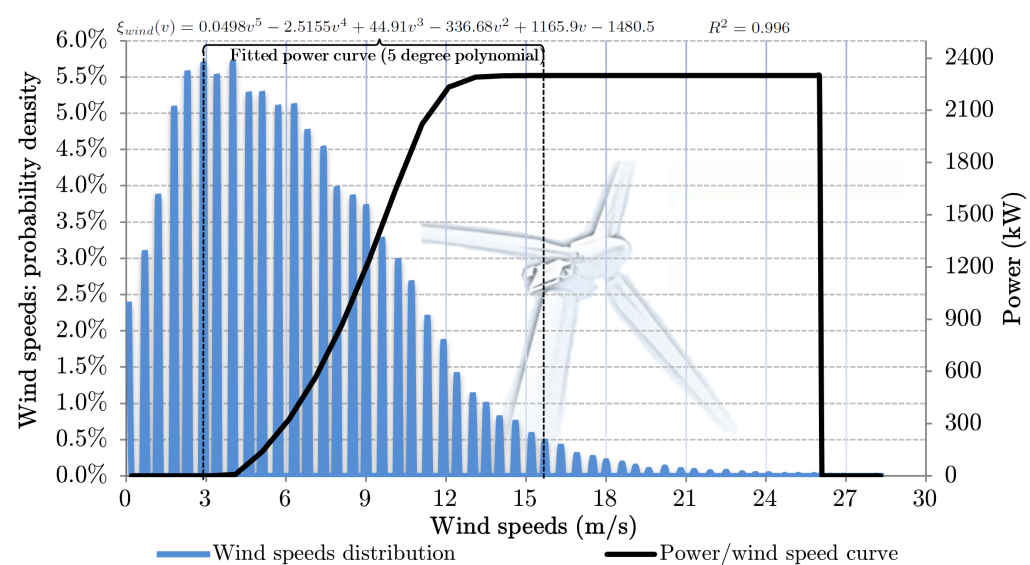

Fig. 2 Wind speed distribution and power curve of a $2.3 \mathrm{MW}$ wind turbine.

- physical operational features also bring a degree of intermittency to wind generation.

It is important to mention that wind is of course not stable over halfhourly intervals. It shifts quickly with very high frequency. However, there is no reason to represent these short-term variations in our wind modelling, since the optimization model would not react to them in any case. Although the short-term variation could be important for engineering aspects of this problem (e.g. reliability and power quality), the value of a battery is more about average values and trends, rather than noise. That is, if we had used finer time steps while modelling wind realizations, in fact representing shortterm variation, the model would in any case only react to half-hourly averages.

\subsection{Medium-size electrical battery}

Storing energy, for a later period, performs either a specific power stability application or an energy balancing operation. In our case, storing energy allows load-levelling by energy arbitrage and smooth wind energy from distributed generation. Additionally, distributed storage could be integrated to ancillary services to support grid operations via demand-side mechanisms.

Energy storage technologies are mainly available in the form of pumped storage. In recent years, however, there have been numerous initiatives and demonstration projects to install medium to large size batteries for different applications (Eyer and Corey 2010; EPRI 2010). According to a database by the United States Department of Energy (2013) there are around 400 electricity storage projects deployed worldwide. Among these case studies and demonstration projects, the vanadium redox flow battery (VRFB) has shown a good level of maturity, operationally speaking, as well as strong prospects on commercial viability (Banham-Hall et al. 2012). In a nutshell, a flow battery is 
an electrochemical electricity storage device, somewhere between a standard rechargeable battery and a fuel cell. For our DG system we consider a VRFB battery similar to the devices utilized and described in the review by Kear et al. (2012) and the United States Department of Energy (2013) examples.

There are many technical aspects that concern the physical operation of an electrical battery which mainly depends upon the storage application context. For our model, we focus on the primary performance indicators for energy storage devices: charging/discharging rates, capacity and efficiency. These are firstly presented in an equality constraint to emulate the basic inventory intratemporal dynamics for each time span $[t-1, t]$. That is to say, the state or level of the battery $S^{(t)}$ at time $t$ is equal to the prior $t-1$ period level of storage $S^{(t-1)}$ plus the storage charge input $B_{\text {in }}^{(t)}$ minus discharge output $B_{\text {out }}^{(t)}$. In short, we have the balancing equation:

$$
\overbrace{S^{(t-1)}+\eta_{\text {eff }} \cdot B_{\text {in }}^{(t)}-B_{\text {out }}^{(t)}}^{\text {Stored prior+Charging - Discharging }}=\overbrace{S^{(t)}}^{\text {Stored at } t}
$$

In this constraint, $\eta_{\text {eff }}$ is the round-trip energy efficiency of the battery. Hence, this energy balance equation determines the battery charging/discharging decisions based on the interactions in eq. (1) and the state of $S^{(t)}$. As a result, charging and discharging capacities (which amount to rates in a time continuous model) are also needed:

$$
\begin{aligned}
& B_{\text {in }}^{(t)} \leq \alpha_{\max } \\
& B_{\text {out }}^{(t)} \leq \beta_{\max }
\end{aligned}
$$

Typically, these rates are functions of the maximum amount of storable energy which we note as the battery capacity $\bar{S}_{\max }$. Moreover, we address the impact of full battery discharge on battery life and efficiency performance by restricting the storage to a minimum $\underline{S}_{\min }$ (known as the depth of discharge).

$$
\underline{S}_{\min } \leq S^{(t)} \leq \bar{S}_{\max }
$$

In general, there is a negative relationship between battery discharge rate and battery efficiency. The higher discharge rate is used, the lower is the battery efficiency. See for example Kear et al. (2012) for a discussion. The battery parameters chosen for this paper are according to the manufacturing specifications by Prudent Energy group (2011). In our case, the VRFB roundtrip efficiency $\left(\eta_{\text {eff }}\right)$ is set to $80 \%$, corresponding to a discharge rate of $250 \mathrm{~kW}^{4}$ for one hour. Also, since efficiency is affected by the depth of discharge of a battery, we set the lower capacity bound $\underline{S}_{\min }$ to $20 \%$ of the rated capacity. So in our discrete model, we have the storage bounds $\bar{S}_{\max }=250 \mathrm{kWh}$ and $\underline{S}_{\min }=50 \mathrm{kWh}$. The discharge capacity is $\beta_{\max }=100 \mathrm{kWh}$ per half-hour time interval, but it can be calibrated or scaled to another discharge time (up to

\footnotetext{
4 Prudent Energy's standard VRB-ESS MW-Class module. This 250KW battery is designed for the support of renewable energy integration for micro grids.
} 
10 hours discharge). Finally, the maximal charge rate under these conditions has a charge/discharge ratio 1.5:1 which is approximately $\alpha_{\max }=65 \mathrm{kWh}$. Note that, to have a fixed $\eta_{\text {eff }}$, we assume an approximate linear relationship between the storage energy level and the charging/discharging rates.

\section{Modelling a stochastic programming framework}

As noted earlier, we propose a multi-stage stochastic programming model to address the treatment of short-term wind uncertainty. We have chosen a stochastic programming framework here, rather than what is possibly more common in a context where we are in fact looking for decision rules, namely stochastic dynamic programming. A detailed discussion of this relationship can be found in Chapter 1 of King and Wallace (2012). Stochastic programming is usually used to model what to do now, based on the present situation and available resources (transient decision making), while dynamic programming is better suited to study decision rules, that is, what to do in all possible situations (steady state decision making). As we shall note in the data analysis later, the time dependency in wind speeds is rather strong within a day. Hence, if dynamic programming was to be used taking this dependency into account, the state space would grow substantially. As a consequence, we have chosen a stochastic programming approach as time dependencies do not increase the computational burden in this framework. Of course, stochastic programming has other challenges, such as the size of the scenario tree, but in this specific setting, this disadvantage was more than offset by the ability to handle complicated time structures.

\subsection{Multi-stage stochastic program}

In our multi-stage model, the half hourly wind energy values $\xi_{\text {wind }}^{(t)}$ are represented through a scenario tree (see Figure 3). Our model contains five stages, $k=1,2, \ldots, K=5$ (though any choice is possible, relative to the solvability of the resulting model). The first stage is shorter than the others, in our case one hour, as we assume that wind speeds are known for that period (so stochastically, we have four stages). Then three stages, each of four hours, follow before we end with a longer stage covering 11 hours. Modelling-wise, decisions are always made in the beginning of each stage - at 8 a.m., 9 a.m., 1 p.m., 5 p.m. and 9 p.m. respectively (see $t \in T_{k}$ in Figure 3 ). In a multi-stage model it is common to design the last stage longer than the others. Typically, the argument is that it is farther into the future (and hence less interesting) or that discounting results in it being less important. In our case, we have modelled this stage longer since it represents the calm period of the day after the evening peak and before the morning peak - when prices are low and stable. In this long stage, the battery is primarily recharged in preparations (energy arbitrage) for the next day morning peak (Sec. 4.4 and 5.2 follows this discussion in detail). 


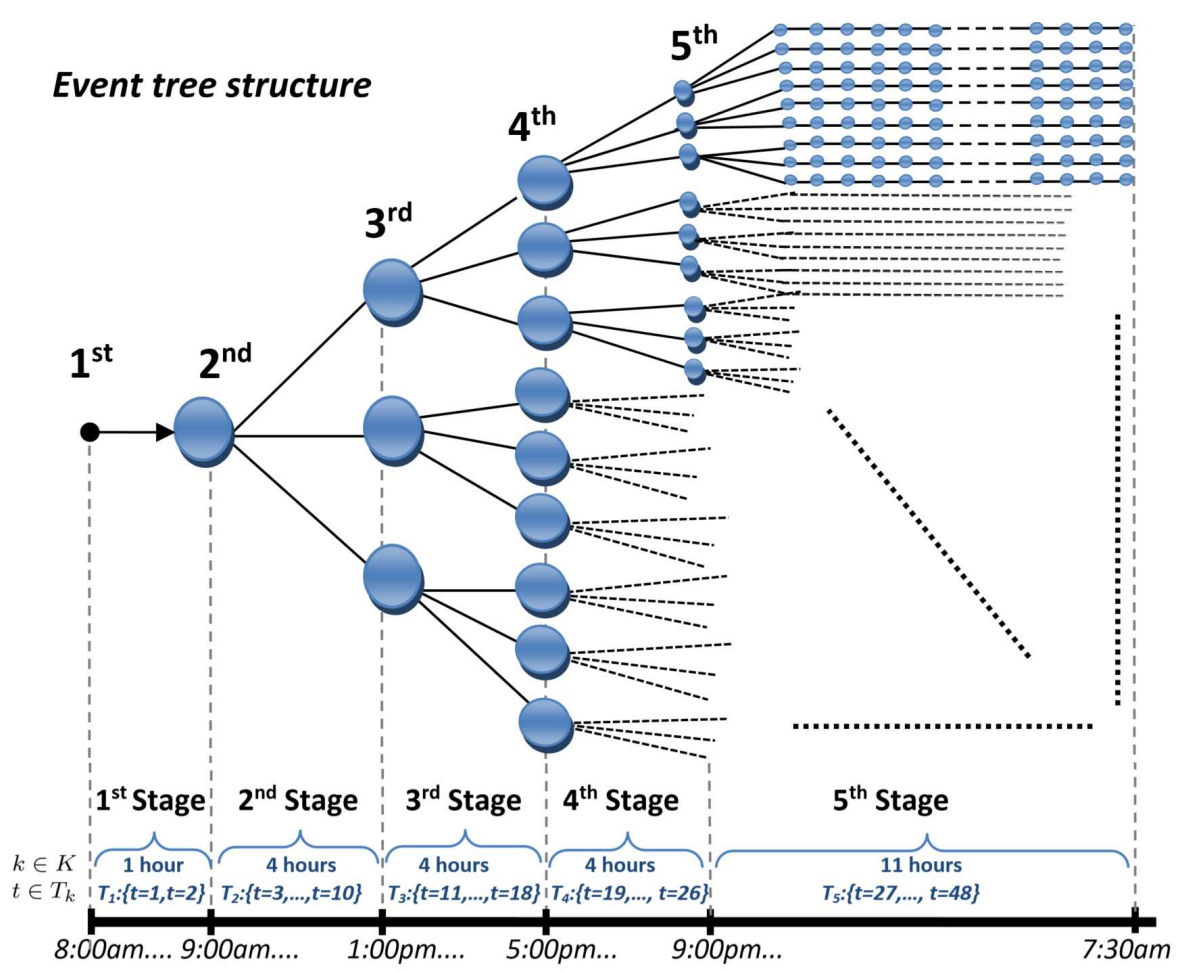

Fig. 3 Illustration of the scenario tree events for wind realizations in one day.

4.2 Characterization of wind speed uncertainty via a scenario tree

In order to characterize wind speed uncertainty, we use historical wind speed data from a meteorological station to create scenarios $\left(\xi_{\text {wind }}^{\left(t, \omega_{k}\right)}\right.$ notation) with corresponding probabilities $\left(\pi_{\omega_{k}}\right)$ for each stage (denoted by $k$ ). We analyse this wind speed data as a univariate stochastic process, and look for a memory structure that represents the underlying dependency in the time series. We do so by applying the ARMA statistical time series models commonly used in the power systems literature to produce scenarios for short-term wind energy uncertainty (see for example Chapter 3 in Conejo et al. 2010).

Specifically, to create ARMA models, we follow the implementation procedure for time series developed by Torres et al. (2005) for short-term wind speeds. The typical autoregressive moving average (ARMA) process applied to wind speeds $\left(v^{(t)}\right)$ is mathematically expressed as:

$$
v^{(t)}=\phi_{1} \cdot v^{(t-1)}+\ldots+\phi_{p} \cdot v^{(t-p)}+\varepsilon^{(t)}+\theta_{1} \cdot \varepsilon^{(t-1)}+\ldots+\theta_{q} \cdot \varepsilon^{(t-q)}
$$

This time series relies on the $p$ auto-regressive (AR) parameters $\phi_{1}, \phi_{2}, \ldots, \phi_{p}$ the $q$ moving averages (MA) $\theta_{1}, \theta_{2}, \ldots, \theta_{q}$ and the error terms $\varepsilon^{(t)}$. We estimate 
(a) Wind speeds error forecast distribution

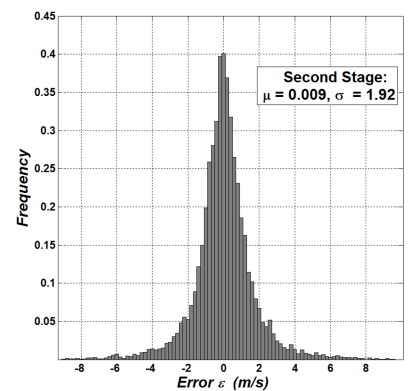

(b) Scenario generation from an $\operatorname{ARMA}(1,4)$ model

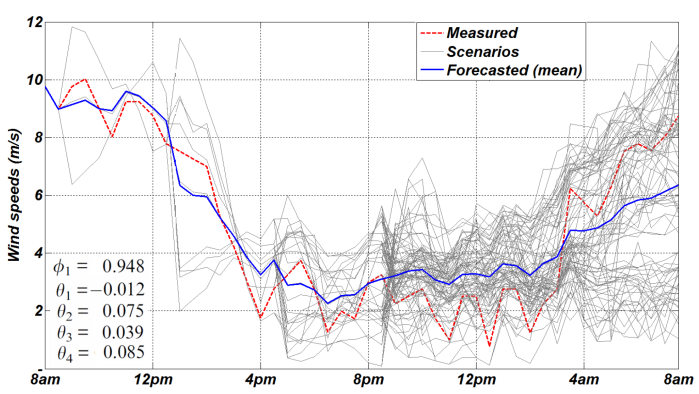

Fig. 4 Historical distribution of actual wind speeds $(\mathrm{m} / \mathrm{s})$ for a four hour period and scenarios generated for the next day.

these parameters by fitting and analysing the historical wind speed data. Then, we forecast the wind speeds of the next stage using forecasting errors to create the scenarios as was done by Schütz and Tomasgard (2011). In fact, Sharma et al. (2013) discuss this methodology specifically for wind speeds in a stochastic programming framework.

In our case, for the second stage $(k=2 ; t=3, \ldots, 10)$ we use the ARMA model to generate the expected (forecasted) wind speed and combine it with two scenarios that approximate the error term $\varepsilon \sim N(0, \sigma)$ by a discrete distribution based on the wind history up to that point. Conditional on each of these wind realizations in the second stage, wind is forecasted for the third stage $(k=3 ; t=11, \ldots, 18)$ with a corresponding branching. The same process is applied to the remaining stages. Hence, from the second stage onward, each data path is generated with the ARMA model based on the wind scenarios of the previous stages. We discretize such that the mean (forecast) is assigned a probability of 0.4 and each of the other two scenarios a probability of 0.3 . In our model formulation, we note the wind scenarios $\xi_{\text {wind }}^{\left(t, \omega_{k}\right)}$ to be indexed by $\omega_{k}$ whom belongs to a particular branching set $\Omega_{k}$ at stage $k$.

Note that the idea in this paper is neither to forecast wind generation for the next day nor to develop a precise short-term wind prediction model. What we are interested in is to produce scenarios that approximate wind behaviour, based on existing literature, so as to get a reasonable scenario framework to estimate the value of electricity storage. In the literature, Weber et al. (2009) and Küchler (2009) used a similar multi-stage approach to model wind speeds with an ARMA time-series. For instance, the left-hand picture in Figure 4 shows the forecasting errors over a four hour period from our case study. This resembles the errors reported by Torres et al. (2005). We use this spread to generate the scenarios as seen in the right-hand picture in Figure 4 for a day in January under an initial ARMA $(1,4)$. 
4.3 Model formulation

The model objective is to determine the optimal operational decisions of the storage so as to minimize the expected grid consumption costs based on demand loads, wind generation availability and price fluctuations over the day (discretized in $30 \mathrm{~min}$. time intervals). Expectation is with respect to the wind scenarios (Figure 3), represented as $\xi_{\text {wind }}^{\left(t, \omega_{k}\right)}$ in our stochastic formulation. Taking into account all the terms and constraints previously described, we consider the following multi-stage stochastic linear program ${ }^{5}$ :

$$
\underset{C_{\text {grid }}, S, B_{\text {in }}, B_{\text {out }}}{\operatorname{Minimize}}\{\overbrace{\sum_{\omega_{k} \in \Omega_{k}} \pi_{\omega_{k}} \sum_{k \in K} \sum_{t \in T_{k}}\left[p_{\text {el }}^{(t)} \cdot C_{\text {grid }}^{\left(t, \omega_{k}\right)}\right]}^{\text {Minimize grid cost consumption }}\}
$$

Subject to:

$$
\begin{aligned}
& \text { for all: } \begin{cases}\xi_{\text {wind }}^{\left(t, \omega_{k}\right)}+C_{\text {grid }}^{\left(t, \omega_{k}\right)}+B_{\text {out }}^{\left(t, \omega_{k}\right)}+L_{\text {base }} \geq d_{\text {load }}^{(t)}+B_{\text {in }}^{\left(t, \omega_{k}\right)} & \text { supply } \geq \text { demand } \\
S^{\left(t-1, \omega_{k}\right)}+\eta_{\text {eff }} \cdot B_{\text {in }}^{\left(t, \omega_{k}\right)}-B_{\text {out }}^{\left(t, \omega_{k}\right)}=S^{\left(t, \omega_{k}\right)} & \text { amount stored } \\
\underline{S}_{\min } \leq S^{\left(t, \omega_{k}\right)} \leq \bar{S}_{\max } & \text { storage capacity }\end{cases} \\
& t \in T_{k} \quad B_{i n}^{\left(t, \omega_{k}\right)} \leq \alpha_{\max } \quad \text { charge rate } \\
& \omega_{k} \in \Omega_{k}\left(B_{\text {out }}^{\left(t, \omega_{k}\right)} \leq \beta_{\max } \quad\right. \text { discharge rate } \\
& C_{\text {grid }}^{\left(t, \omega_{k}\right)}, S^{\left(t, \omega_{k}\right)}, B_{\text {in }}^{\left(t, \omega_{k}\right)}, B_{\text {out }}^{\left(t, \omega_{k}\right)} \geq 0 \quad \text { non-negativity }
\end{aligned}
$$

Note that some approximations and assumptions have been made in the model to make it numerically tractable as well as to remove unessential technical details. First, as previously discussed, the physics of energy storage in terms of the relationship (linear) between charge/discharge rates and storage capacity. Second, the above formulation may lead to simultaneous charging and discharging. This may result in a lack of uniqueness in the solution in cases where wind energy surpasses demand for an extended period of time. This is one situation in which the model wastes energy; however, this does not affect the value of energy storage. Third, the base load production $L_{\text {base }}$ is treated as a constant. For our DG case study this production stems from a CHP running at full capacity in winter and not at all in summer. The point is that the CHP decisions have a longer time frame than one day.

\subsection{End storage level (tail effects)}

The model in (6) is designed for 48 time intervals, i. e. 24 hours. And the goal of the model is to understand the value of the battery, not to provide

\footnotetext{
5 Note that the solution lives on a scenario tree like the one in Figure 3.
} 
operational details for controlling the charging and discharging policies over time. For an operational model, more technical engineer details would have to be included. So, on one hand this is a strategic model, on the other hand, it must contain enough operational details to understand the effects of the strategic decision of installing a battery of a certain capacity.

The main challenge, which is typical of all finite horizon models for (principally) infinite horizon problems, is that of handling end effects. Here, the issue is deciding on a reasonable amount of energy to start and end the storage levels, i. e. $S^{\left(t=1, \omega_{1}\right)}$ and $S^{\left(t=48, \omega_{5}\right)}$. In the context of our case study, a university campus with a installed generation capacity well below its base load and with no possibility of delivering to the grid, we have made the following assumption: before the morning peak, the battery is fully charged (either from wind or from cheap night-time electricity from the grid) and ready to discharge during the high price period. As a result, in our model we start our planning day at 8 a.m. with a full battery, and end at 8 a.m. the next day, again requiring a full battery. See the time-line in Figure 3 for an illustration. Other starting times were tested to corroborate that $8 \mathrm{a} . \mathrm{m}$. is the appropriate time to start the model calculations under this assumption. This is a distinction to other studies, for example Vespucci et al. (2010) who defined the lower bound for the last period to be half of the initial level.

This tail effect modelling might misrepresent special situations when wind exceeds local demand. If wind exceeds demand after the morning peak, our starting storage level assumption holds and the wind surplus is properly utilized. However in cases where wind exceeds demand during morning peak, the model to some extent becomes illogical: a wind surplus should have been absorbed by the battery (which is already full) instead of being wasted. We find this acceptable since we are concerned with sites that use wind turbines and batteries in a local grid for internal consumption (not for delivery to the national grid), and where it is unusual that a wind turbine, even if it delivers at installed capacity, will deliver more than the morning peak demand. If a site invests in higher levels of wind capacity, one would expect a similar investment in infrastructure to deliver to the grid.

\section{Case study: university campus energy system}

In the preceding sections we discussed the typical profile of a large end-user with a local DG system followed by its stochastic programming formulation. We now highlight the main features of a case study set up for Lancaster University campus (see Lancaster University 2011 for more details). This case study consist of the following specifications:

- Annual campus electricity consumption in 2010 was around 33GWh with a registered maximum peak load of $3.1 \mathrm{MW}$ (in a half hour period).

- The campus is composed of a mix of residential, office and commercial buildings and has a total population of around 15,000 people. 
- A 2.3MW wind turbine (Figure 2) is currently operational with an expected annual contribution of $14-17 \%$ to local demand.

- A gas fired CHP unit is used as base load for heating and electricity demand. It has a maximum electricity output of $2 \mathrm{MW}\left(L_{\text {base }}\right)$. The CHP is only operational in winter or cold periods (around $50 \%$ of the year).

- The university campus controls autonomously its own micro grid through a high voltage ring circuit composed of 13 high voltage $(11 \mathrm{kV})$ substations which are stepped down to $400 \mathrm{~V}$ for the end-user.

- The grid connection has a total capacity of 10.5 MVA supplied through two $11 \mathrm{kV}$ and one $33 \mathrm{kV}$ underground high voltage cables.

- For each building on campus a smart meter program exists complemented with other low-carbon and energy efficiency measures.

- No wind surplus is being sold back to the grid resulting in wind curtailments when it exceeds local demand.

\subsection{Model implementation}

The model was validated by using actual data sets for energy demand, electricity prices and wind speeds. Our model determined minimal energy costs over the course of a year divided into individual optimization problems for each day. Additional to the 2010 half-hourly electricity demand data provided by Lancaster University, we gathered the following data sets:

- Wholesale spot prices were collected from ELEXON-Ltd (the UK market regulator) for all 2010. We used the reference price data (RPD) index as it reflects the price of wholesale electricity in the short-term market. These electricity prices are comparable to day-ahead market prices and are assumed to be known for the next 24 hours (deterministic in nature).

- Wind scenarios were generated from historical wind speed meteorological data collected ${ }^{6}$ by Hazelrigg Weather Station - the site of the university wind turbine. The $\operatorname{ARMA}(p, q)$ models fitted to the data exhibited a range of MA values when $2 \leq q \leq 6$. This showed a steady dependency for contiguous stages in the model as shown on the example of Figure $4 b$. The overall annual root mean square errors for the last four stages were: 1.8 $\mathrm{m} / \mathrm{s}, 2.3 \mathrm{~m} / \mathrm{s}, 2,6 \mathrm{~m} / \mathrm{s}$ and $3.1 \mathrm{~m} / \mathrm{s}$ respectively (refer to Torres et al. 2005, for details on similar results and discussion).

As discussed earlier, by analysing historical wind speed data for the Lancaster region we divided our planning horizon in 5 stages. Figure 3 shows the five-stage scenario tree designed for the day-ahead operational planning. In this tree, three branches leave each node to represent wind realizations, resulting in a total of 81 scenarios. As noted in the figure's time-line, a perfect forecast is assumed for the first stage which is composed of two time periods (one hour). The next three stages consist of four hours each. Stage 5 contains

6 The wind speeds data stem from an anemometer height lower than the wind turbine height. To account for this difference, we follow a process noted in Sharma et al. 2013. 
the remaining 11 hours (22 periods). As outlined before, this is done because the night is a calm period with stable low prices and demand (see the dynamics in Figure 5). Regarding the number of scenarios choice, we carefully selected a computationally tractable scenario tree size that is large enough to support a sensible and stable solution. For this, we refer to Bruninx et al. (2014) and Vespucci et al. (2010) on a validation of different number of wind scenarios in which stability is illustrated around 50 to 100 scenarios.

The described optimization framework was implemented in MATLAB (Mathworks 2012) for data processing and scenario generation while a Python environment was used to set up the problem formulation, using cplex (IBM 2012) to solve the problem. Performing these calculations in a regular laptop takes 2 minutes for each optimization (one day) and up to 12 hours for the whole year (365 optimization instances). The dimension of the problem consist of 8672 variables and 4336 constraints for each optimization problem.

\subsection{An illustrative example}

Computational results corresponding to three optimization instances are exhibited in Figure 5. To better comprehend the different dynamics in the energy system, this example exhibits a case with perfect foresight on wind energy (i.e deterministic case). We shall later turn to the stochastic cases as they are what we actually study in this paper. The data used for this example represents a Thursday, Friday and Saturday in September 2010. For each of the three 48-period (24-hour) optimization problems, the actual recorded halfhourly electricity demand is used. The wind energy is calculated from wind speed scenarios using the power curve function in Figure 2. Lastly, the market regulator spot reference prices for the same three days are used as proxies for energy costs (black line in Figure $5 a$ ). The storage parameters are set to the baseline battery module noted in Sec. 3.3. However, these are scaled up to the case study context by considering a $\bar{S}_{\max }=4 \mathrm{MWh}$. Summarizing the observation of this three-day example we highlight the following:

- Prices: According to the National Grid, peak time for electricity prices is officially defined as the 7 a.m. to 7 p.m. time span. The black line of Figure $5 a$ shows two main peak times in the day, morning ( 8 a.m. to noon) and late afternoon (5-9 p.m.). Non-peak time exhibits almost no price jumps this being the time for energy storage arbitrage operations - charging the battery to avoid future high prices. This reinforces the argument that the model's most critical economic decisions are in stages 1 to 4 (daytime).

- Demand: In the same chart (Figure $5 a$ ), the university campus load presents a bell shaped demand pattern with a load surge around 7-9 a.m. and a decline after office hours (6 p.m.). The total demand of these 3 days is 290MWh. The CHP contributes $50 \%$ of the demand, while wind and grid inputs cover $22 \%$ and $24 \%$ respectively. The battery discharge satisfies the remaining $4 \%$. 
(a) Supply-Demand results and prices
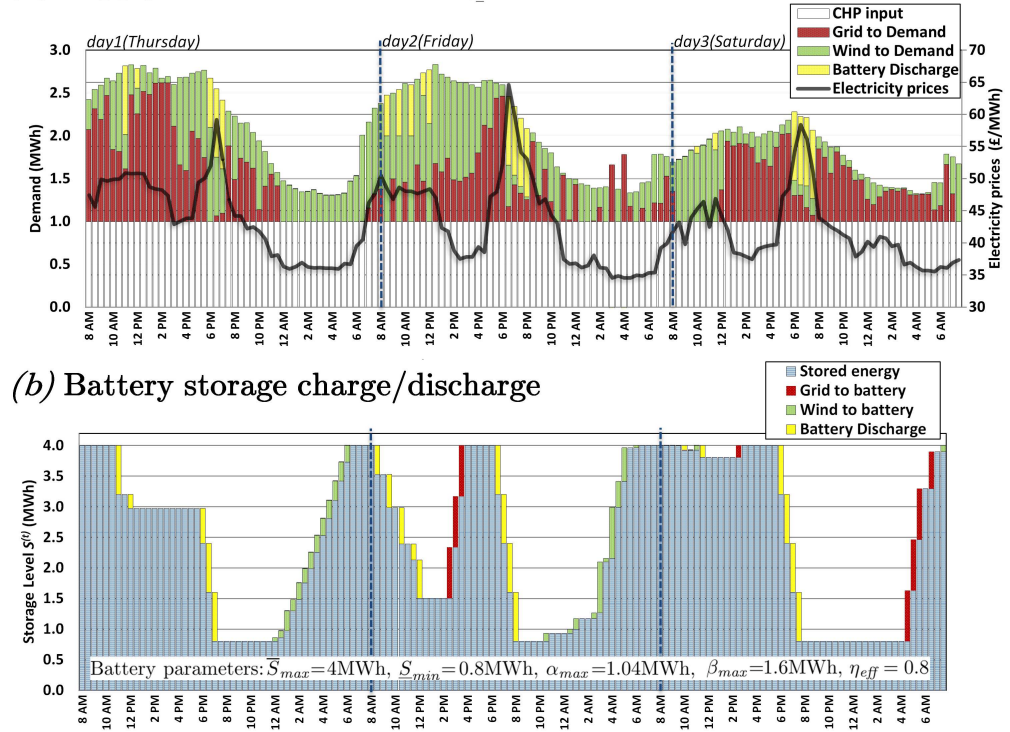

(c) Wind generation output

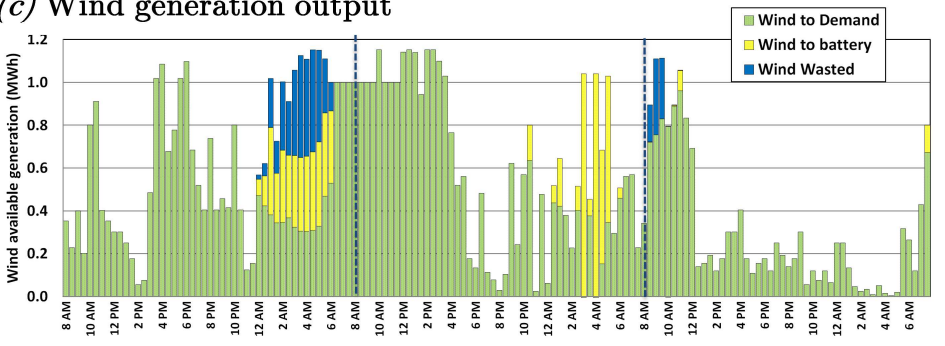

Fig. 5 Example of model results for three continuous optimization instances, each one day long (discretized in $30 \mathrm{~min}$ intervals $[t-1, t]$ ).

- Battery: Clearly storage decisions are driven by the opportunity to avoid high electricity prices, as well as to save wind surplus (see Figure $5 b$ ). For instance, as the day(s) roll over, a clear load shifting occurs on the 6-8 p.m. peak time on Saturday (day 3) as well as in the morning peak for Friday (day 2), while wind leftover is saved during the night for Thursday and Friday. For this particular example, the battery charging inputs $B_{\text {in }}$ came from the grid (47\%) and wind energy (53\%). Also, it can be observed that the tail effect assumption of a full battery in the early morning holds.

- Wind: Figure $5 c$ shows the intermittent nature of wind energy. Observe that the model decision is to take up all the available wind to satisfy demand. In off-peak time periods, since wind energy might exceed demand (see the first two days 12-6 a.m. period), the surplus is saved into the battery. If the battery is fully charged or its charging capacity exceeded, wind energy is wasted as shown on the first day. In this three day example, 
$6 \%$ of wind generation is wasted. Running the same case, but without a battery, the curtailment increases to $15 \%$.

In short, for this particular example, the battery presence in the energy system saves costs in the range of $5 \%$ (compared to not having the battery). As illustrated, this cost saving is closely related to the level of local generation $\left(\xi_{\text {wind }}^{(t)}+L_{\text {base }}\right)$. For instance, if the CHP is not operational (e.g. summer) the value of energy storage in this example drops to $2 \%$ as no wind smoothing operations occur (demand always above supply). Given that the key drivers for energy storage operations are energy arbitrage and the smoothing of renewable output, a wind-plus-battery combo exhibits a higher overall value.

\subsection{The value of energy storage}

We now turn to the stochastic case, where all runs of the model are based on our stochastic programming formulation. To investigate the effect of wind uncertainty, we consider four battery capacities $\left(\bar{S}_{\max }=0,2,4,8 \mathrm{MWh}\right)$ along with different wind/base-load combinations. This analysis required running the model for 365 days on our 2010 data sets. Columns in Figure 6 depict the estimated total annual cost savings for the expected value problem (using only forecasted wind), compared with the annual saving using the stochastic programming model. These savings represent the gains of installing a battery, also noted as \% cost savings. That is, the difference between the annual cost of a battery Case and the value of its equivalent problem without storage (same wind/CHP combo size but $\bar{S}_{\max }=0$; see Table 2 for details). The pie chart $^{7}$ for each battery size represents the proportion of battery charge (i. e. $B_{i n}^{\left(t, \omega_{k}\right)}$ ) that came from the grid (arbitrage value) and from smoothing wind (wind value). From these results we highlight the following:

- One wind turbine: Case I represents the current DG units operational in our case study. They satisfy $40 \%$ of the annual demand. Throughout the year, this combined generation exceeds demand $\left(\xi_{\text {wind }}^{(t)}+L_{\text {base }} \geq d_{\text {load }}^{(t)}\right)$ around $4 \%$ of the time. Consequently, arbitrage drives energy storage operations, with $91 \%$ of battery charge coming from the grid and $9 \%$ from excess wind (2MWh battery pie chart). Its counterpart case with no CHP (Case II) always has supply below demand, so storage is only charged by the grid (100\% arbitrage pie charts). Observe that since wind uncertainty is not a main driver behind storage decisions, there is no significant difference between the stochastic and deterministic solutions. Therefore, in these cases, capturing wind uncertainty is not significant for the valuation of batteries.

\footnotetext{
7 Due to the complexities associated with the calculations of the multi-stage stochastic setting, the pie charts numbers come from the expected cases. The idea is to simply illustrate and qualitatively note that 'wind value \%' causes uncertainty on storage decisions.
} 

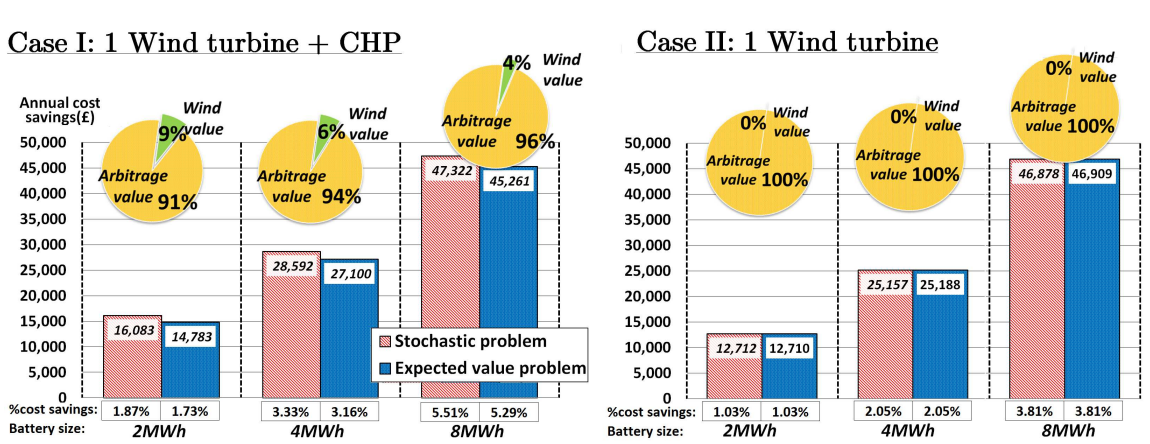

Case III:2 Wind turbines + CHP(base load)
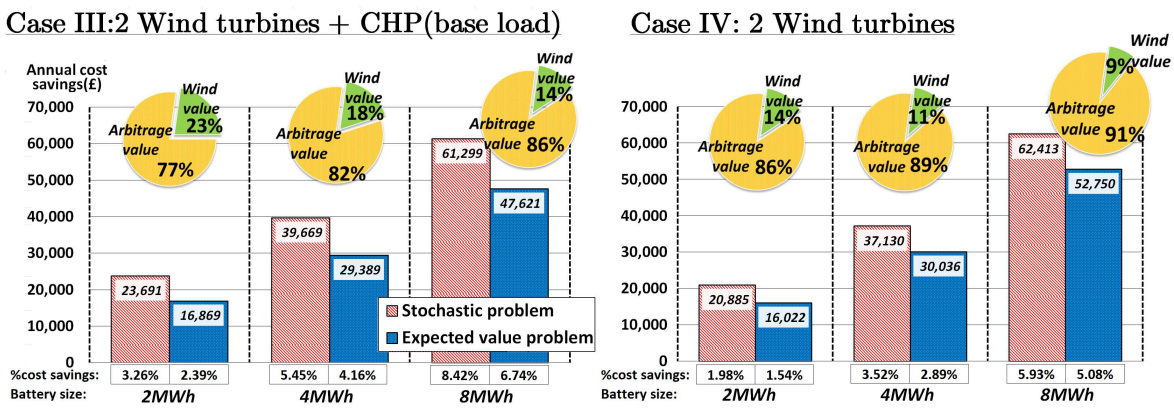

Case V: 3 Wind turbines + CHP(base load)

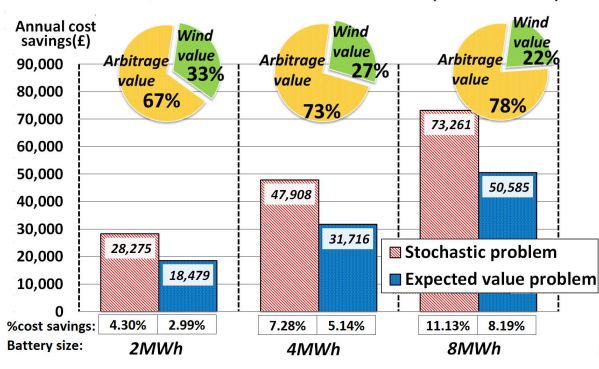

Case VI: 3 Wind turbines

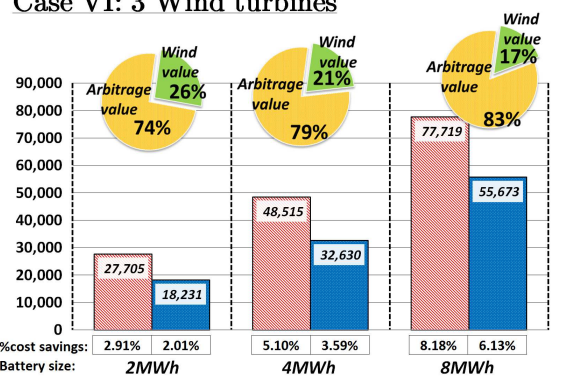

Fig. 6 Annual cost savings of energy storage for different unit size combinations for installing a battery.

- Multiple wind turbines: Clearly for Cases III and IV, the more supply surpasses demand throughout the day, the more valuable the battery operations become. For instance, the increasing wind contribution to the value of storage (noted in the pie charts), derives mainly from the early morning surplus discussed in Figure 5. This value added from wind smoothing becomes evident if we compare the storage value of Case II (2MWh) in which we have a gain of $£ 12,712$ (only from arbitrage) with the gains of $£ 20,885$ and $£ 27,705$ for Case IV and VI respectively. Also, we see that the differences between the stochastic and deterministic valuations accentuate since uncertainty becomes more prevalent in the storage decisions. 
5.4 The importance of considering wind uncertainty

Since uncertainty becomes more relevant when larger wind generation capacities are in place, here we focus on the specific cases of having two and three wind turbines (case III to VI, Figure 6). The consistent differences in valuation between the stochastic and expected problems come from the limitations of not identifying strategic options in the expected case. For instance, when using a $2 \mathrm{MWh}$ battery (Case VI) the expected case (deterministic model) underestimates the value of the battery by $50 \%$ compared with the stochastic case. Similar situations occur for other battery sizes in which the stochastic solution provides a consistent improvement compared with its deterministic counterpart, e.g., $40 \%$ for $2 \mathrm{MWh}$ on Case III, $30 \%$ for $2 \mathrm{MWh}$ on Case IV, $53 \%$ for $2 \mathrm{MWh}$ on Case $\mathrm{V}$, etc.

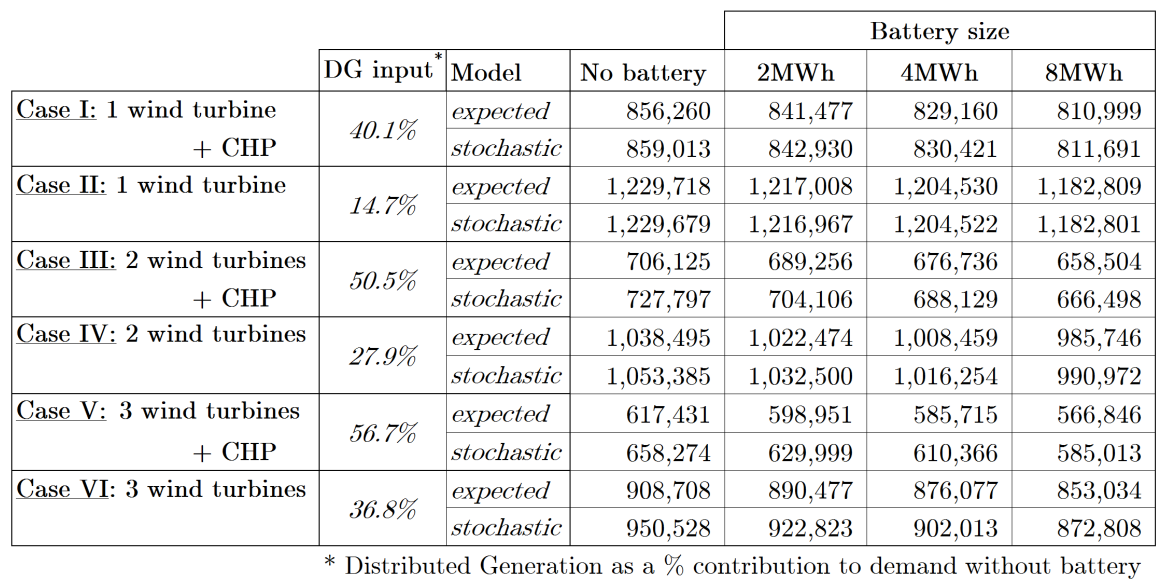

Table 2 Total annual costs for each case unit mix composition in GBP (£) presented in Figure 6.

We refer to this underestimation of the value of the battery as the error of not considering uncertainty, and not as the value of the stochastic solution (VSS). To be sure, we are not measuring how well a deterministic solution behaves in a stochastic environment (the purpose of VSS), but rather how accurate a value estimation based on a deterministic model is relative to a more appropriate stochastic model. That is, we are asking if an appropriate strategic decision can be made based on a deterministic model. Observing how imprecise we were by estimating the value of the battery with a deterministic model has important implications on the strategic decision of whether or not storage might be worth installing. An increase by $50 \%$ in these savings may indeed facilitate reaching the break-even point for investment decisions in end-user energy storage. As this large end-user is not delivering to the grid, for moderate 
situations (Case I and II) using the deterministic model seems reasonable. However the error should not be neglected when larger units (i.e. DG/battery combos) are considered.

\section{Conclusion and perspectives}

In a smart grid setting, this paper presented a model to valuate distributed electricity storage from the viewpoint of a large end-user. We investigated how energy storage reduces costs for the site by matching the DG intermittent renewable output with the customer demand. Our analysis focused on the effect of wind uncertainty on the strategic valuation of energy storage. Attention was paid to the level of DG participation (wind surplus) along with the role of energy arbitrage operations. In a numerical study, we observed that the value of energy storage for the end-user is a reduction of up to $10 \%$ of their energy costs. We showed that the storage value is dependent on the unit's renewable generation capacity (DG/battery combo). But centrally to this paper's key question, we found that the error of not considering wind uncertainty (deterministic case) causes an underestimation of the value of storage by $30 \%$ for two wind turbines and up to $50 \%$ for three wind turbines. As a consequence, basing strategic investment decisions on deterministic valuation models can lead to largely imprecise estimates and an inaccurate valuation judgement.

As the prospects and development of storage technologies continue to mature, direction for further research should take into account additional aspects when valuing energy storage, such as:

- Assess the effects of randomness in grid prices on the valuation of electricity storage. We have left this out here since, for our case study (and for many other electricity markets), prices are not random over a period of 24 hours. However, we plan to conduct later studies where also grid prices are uncertain.

- Estimate the benefits of electricity storage to the local distribution network or utility. Customer-sited storage reduces maximal demand which can save investments on distribution requirements. Also, the efficient utilization of local DG can save on transmission and distribution losses.

- Develop business models to investigate storage ownership that captures a storage value favourable to the local utility, the grid and the end-user. For instance, consider modelling other grid features to reflect operations on ancillary services and power quality issues.

- Model the economic implications of integrating end-user energy storage to electricity markets. A broad deployment of end-user electricity storage would impact wholesale electricity prices and create value for a more efficient grid supply-demand equilibrium.

Acknowledgements We thank the Facilities Department at Lancaster University for providing information on the campus energy system as well as electricity demand data. Likewise, to the Lancaster Environmental Center for sharing high frequency data on historical 
wind speeds. Lastly, our acknowledgements to the Department of Management Science at Lancaster University and the Department of Business and Management Science at The Norwegian School of Economics for facilitating research visits.

\section{References}

Ahlert, K. and van Dinther, C. (2009). Sensitivity analysis of the economic benefits from electricity storage at the end consumer level. In PowerTech, 2009 IEEE Bucharest, pages 1-8.

Banham-Hall, D., Taylor, G., Smith, C., and Irving, M. (2012). Flow batteries for enhancing wind power integration. Power Systems, IEEE Transactions on, 27(3):1690-1697.

Brown, P., Lopes, J. P., and Matos, M. (2008). Optimization of pumped storage capacity in an isolated power system with large renewable penetration. IEEE Transactions on Power Systems, 23.

Bruninx, K., Delarue, E., and Dhaeseleer, W. (2014). A modeling framework for the integration of intermittent renewables: stochastic unit commitment. TME Working paper, WP EN2014-1, KU Leuven Energy Institute.

Carbon Connect (2012). Distributed generation: From cinderella to centre stage. Technical report, Policy Connect.

Cardoso, G., Stadler, M., Siddiqui, A., Marnay, C., DeForest, N., BarbosaPvoa, A., and Ferro, P. (2013). Microgrid reliability modeling and battery scheduling using stochastic linear programming. Electric Power Systems Research, 103(0):61 - 69 .

Castronuovo, E. D. and Lopes, J. A. P. (2004). Optimal operation and hydro storage sizing of a wind hydro power plant. International Journal of Electrical Power \& Energy Systems, 26(10):771 - 778.

Conejo, A. J., Carrin, M., and Morales, J. M. (2010). Decision making under uncertainty in Electricity Markets. International Series in Operations Research \& Management Science. New York, Springer.

Crespo Del Granado, P., Wallace, S. W., and Pang, Z. (2014). The value of electricity storage in domestic homes: a smart grid perspective. Energy Systems, 5(2):211-232.

DECC (2013). UK Renewable Energy Roadmap update 2013. Technical report, Department of Energy and Climate Change.

ELEXON-Ltd (2010-2011). The balancing mechanism reporting system, the new electricity trading arrangements, electricity historic prices. [Online] Available: http://www . bmreports.com.

EPRI (2010). Electricity Energy Storage Technology Options: A White Paper Primer on Applications, Costs, and Benefits. Technical report, Electric Power Research Institute.

European Commission (2014). A policy framework for climate and energy in the period from 2020 to 2030. Technical report, Eurpean Union.

Eyer, J. and Corey, G. (2010). Energy storage for the electricity grid: Benefits and market potential assessment guide. Technical report, Sandia National Laboratories. 
Garcia-Gonzalez, J., Ruiz de la Muela, R., Santos, L., and Gonzalez, A. (2008). Stochastic joint optimization of wind generation and pumped-storage units in an electricity market. Power Systems, IEEE Transactions on, 23(2):460 468.

Hanna, R., Kleissl, J., Nottrott, A., and Ferry, M. (2014). Energy dispatch schedule optimization for demand charge reduction using a photovoltaicbattery storage system with solar forecasting. Solar Energy, 103(0):269 287.

Harsha, P. and Dahleh, M. (2015). Optimal management and sizing of energy storage under dynamic pricing for the efficient integration of renewable energy. Power Systems, IEEE Transactions on, 30(3):1164-1181.

Hatziargyriou, N., Asano, H., Iravani, R., and Marnay, C. (2007). Microgrids. Power and Energy Magazine, IEEE, 5(4):78-94.

Hazelrigg Weather Station (2006-2011). Historical wind speed data sets on half hour basis. Further details available at: http: //www.es. lancs.ac.uk/ Hazelrigg/,Lancaster University Enviromental Center.

He, X., Delarue, E., D'Haeseleer, W., and Glachant, J.-M. (2011). A novel business model for aggregating the values of electricity storage. Energy Policy, 39(3):1575 - 1585 .

Hittinger, E., Whitacre, J., and Apt, J. (2012). What properties of grid energy storage are most valuable? Journal of Power Sources, 206(0):436 - 449.

IBM (2012). ILOG CPLEX (Version 12). [Online] Available: http://www . ibm.com/developerworks/downloads/ws/ilogcplex/. [Software].

Kear, G., Shah, A. A., and Walsh, F. C. (2012). Development of the allvanadium redox flow battery for energy storage: a review of technological, financial and policy aspects. International Journal of Energy Research, 36(11):1105-1120.

Kim, J. H. and Powell, W. B. (2011). Optimal energy commitments with storage and intermittent supply. Operations Research, 59(6):1347-1360.

King, A. J. and Wallace, S. W. (2012). Modeling with Stochastic Programming. Springer Series in Operations Research and Financial Engineering. Springer.

Korpaas, M., Holen, A. T., and Hildrum, R. (2003). Operation and sizing of energy storage for wind power plants in a market system. International Journal of Electrical Power and Energy Systems, 25(8):599-606.

Küchler, C. (2009). Stability, Approximation, and Decomposition in Twoand Multistage Stochastic Programming. Stochastic Programming. Springer Verlag NY.

Lancaster University (2011). Lancaster University carbon management plan 2009-2012. Technical report, Lancaster University Facilities Department.

Mathworks (2012). MATLAB R2012b (Version 8). [Online] Available: http: //www . mathworks.com/products/matlab/. [Software].

Mishra, A., Irwin, D., Shenoy, P., Kurose, J., and Zhu, T. (2012). Smartcharge: cutting the electricity bill in smart homes with energy storage. In Proceedings of the 3rd International Conference on Future Energy Systems, eEnergy, pages 29:1-29:10. ACM. 
Mokrian, P. and Stephen, M. (2006). A stochastic programming framework for the valuation of electricity storage. In The 26th USAEE/IAEE North American Conference, Ann Arbor, Michigan, USA, pages $24-26$.

Moreno, R., Moreira, R., and Strbac, G. (2015). A MILP model for optimising multi-service portfolios of distributed energy storage. Applied Energy, $137(0): 554-566$.

Nyamdash, B., Denny, E., and OMalley, M. (2010). The viability of balancing wind generation with large scale energy storage. Energy Policy, 38(11):7200 -7208 .

Prudent Energy group (2011). Storage for a sustaniable future, product brochure. [Online] Available: http://www.pdenergy.com.

Roberts, B. and Sandberg, C. (2011). The role of energy storage in development of smart grids. Proceedings of the IEEE, 99(6):1139-1144.

Schütz, P. and Tomasgard, A. (2011). The impact of flexibility on operational supply chain planning. International Journal of Production Economics, 134(2):300-311. 'Robust Supply Chain Management'.

Scott, W. and Powell, W. (2012). Approximate dynamic programming for energy storage with new results on instrumental variables and projected bellman errors. Working paper, Princeton University.

Sharma, K. C., Jain, P., and Bhakar, R. (2013). Wind power scenario generation and reduction in stochastic programming framework. Electric Power Components and Systems, 41(3):271-285.

Sioshansi, R., Denholm, P., Jenkin, T., and Weiss, J. (2009). Estimating the value of electricity storage in PJM: Arbitrage and some welfare effects. Energy Economics, 31(2):269 - 277.

Sundararagavan, S. and Baker, E. (2012). Evaluating energy storage technologies for wind power integration. Solar Energy, 86(9):2707 - 2717.

Teh, N. J., Goujon, G., Bortuzzo, G., and Rhodes, A. (2011). UK Smart Grid Capabilities Development Programme. Technical report, Energy Generation and Supply, Knowledge Transfer Network.

Torres, J., Garca, A., Blas, M. D., and Francisco, A. D. (2005). Forecast of hourly average wind speed with ARMA models in Navarre (spain). Solar Energy, 79(1):65 - 77 .

United States Department of Energy (2013). DOE international energy storage database. [Online] Available:http://www.energystorageexchange.org.

Vespucci, M. T., Maggioni, F., Bertocchi, M. I., and Innorta, M. (2010). A stochastic model for the daily coordination of pumped storage hydro plants and wind power plants. Annals of Operations Research, 193.

Walawalkar, R., Apt, J., and Mancini, R. (2007). Economics of electric energy storage for energy arbitrage and regulation in new york. Energy Policy, $\mathbf{3 5}(4): 2558-2568$.

Weber, C., Meibom, P., Barth, R., and Brand, H. (2009). Wilmar: A stochastic programming tool to analyze the large-scale integration of wind energy. In Kallrath, J., Pardalos, P., Rebennack, S., and Scheidt, M., editors, Optimization in the Energy Industry, Energy Systems, pages 437-458. Springer Berlin Heidelberg. 